\title{
Network Analysis of COVID-19 Vaccine Misinformation on Social Media
}

\author{
Chad MELTONa, a, , Olufunto A. OLUSANYA ${ }^{\mathrm{a}}$ and Arash SHABAN-NEJAD ${ }^{\mathrm{a}, \mathrm{b}, 1}$ \\ ${ }^{a}$ University of Tennessee Health Science Center - Oak Ridge National Laboratory \\ (UTHSC-ORNL) Center for Biomedical Informatics, Department of Pediatrics, \\ Memphis, TN, USA \\ ${ }^{b}$ The Bredesen Center, University of Tennessee, Knoxville, TN, USA
}

\begin{abstract}
Almost half of the world population has received at least one dose of vaccine against the COVID-19 virus. However, vaccine hesitancy amongst certain populations is driving new waves of infections at alarming rates. The popularity of online social media platforms attracts supporters of the anti-vaccination movement who spread misinformation about vaccine safety and effectiveness. We conducted a semantic network analysis to explore and analyze COVID-19 vaccine misinformation on the Reddit social media platform.
\end{abstract}

Keywords. Vaccines, vaccine hesitancy, social media, misinformation, semantic analysis

\section{Introduction}

Though social distancing, mask usage, and lockdowns help slow the spread of the COVID-19 virus, there is an overwhelming consensus that vaccinations save lives and remain the best defense against new COVID-19 infections. A plague in itself, the spread of vaccine misinformation and disinformation ultimately lead to unquantifiable negative outcomes (e.g., low vaccination rates, increased infectivity, and hospitalization rates, as well as morbidity, and mortality) from vaccine-preventable diseases [1,2].

\section{Methods}

We conducted a semantic network analysis [3] on 5,106 posts displayed by 3,770 unique authors within the Reddit community, r/NoNewNormal from November 23, 2020, to July 27, 2021. Our graph was created based on the calculation of Eigen centrality (EC) between nodes. EC quantifies and measures the specific influence of a node based on the number of edges it shares with other nodes (i.e., influence) [4].

\footnotetext{
${ }^{1}$ Corresponding Authors, Chad Melton (Email: chadmeltone@gmail.com) and Arash Shaban-Nejad (Email: ashabann@uthsc.edu), Centre for Biomedical Informatics, 50 N. Dunlap St., Memphis, TN 38103, USA.
} 


\section{Results}

The EC network rendered with its central hub as being the word vaccine and reported a centrality value of approximately 0.517 . A secondary hub, people, was located relatively close to the main hub. This was somewhat expected due to the querying process while harvesting the data. EC values in our graph ranged from [0.002 survival, 0.517 vaccine]. The distribution of EC values appeared to be Gaussian with a group of four outliers ranging from [0.434, 0.517]. Some subnetworks revealed connections between nodes that could potentially indicate misinformation or hesitancy. The node effects were connected to several other nodes including long, term, vaccine, people, covid, and vaccines. Upon inspection of our data set, we observed clear detection of a vaccinehesitant comment related to this network ("I absolutely will not take it and my wife won't either").

\section{Discussion and Conclusion}

As Proof of Concept, our results suggest that semantic network analysis could be implemented in detecting vaccine misinformation or vaccine hesitancy. Because the causes of vaccine hesitancy are multifaceted, digital intervention technologies should be adopted to surveil and recognize the type of hesitant behavior so an appropriate response can be implemented. These results accompany ongoing research focused on detecting and categorizing vaccine misinformation in social media and other public forums [5]. The information gained from network analysis could be especially useful in designing custom digital technologies $[6,7]$ to educate vaccine-hesitant users or combat outright dangerous misinformation designed to dissuade users from being vaccinated.

\section{References}

[1] Zhang J, Featherstone JD, Calabrese C, and Wojcieszak M. Effects of fact-checking social media vaccine misinformation on attitudes toward vaccines. Preventive Medicine. 2021;145:106408.

[2] van der Linden S, Sander, Graham Dixon, Chris Clarke, and John Cook. Inoculating against COVID-19 vaccine misinformation. EClinicalMedicine 2021;33:100772.

[3] Shin EK, and Shaban-Nejad A. Applied Network Science for Relational Chronic Disease Surveillance. Stud Health Technol Inform. 2019;262:336-339. doi: 10.3233/SHTI190087.

[4] Developers, NetworkX. "NetworkX documentation." Release 1.7, (2012). Available online at: https://networkx.org/documentation/networkx-1.7/index.htmlRelease

[5] Melton, CA, Olusanya OA., Ammar N, and Shaban-Nejad A. Public Sentiment and Topic Modeling Regarding COVID-19 Vaccines on Reddit Social Media Platform: A Call to Action For Strengthening Vaccine Confidence. Journal of Infection and Public Health. 2021;S1876-0341(21)00228-8. doi: 10.1016/j.jiph.2021.08.010

[6] Olusanya OA, Ammar N, Davis RL, Bednarczyk RA, and Shaban-Nejad A. A Digital Personal Health Library for Enabling Precision Health Promotion to Prevent Human Papilloma Virus-Associated Cancers. Front. Digit. Health, 21 July 2021. doi:10.3389/fdgth.2021.683161

[7] Ammar N, Bailey JE, Davis RL, Shaban-Nejad A. The Personal Health Library: A Single Point of Secure Access to Patient Digital Health Information. Stud Health Technol Inform. 2020 Jun 16;270:448-452. doi: $10.3233 /$ SHTI200200. 